\title{
Consideration of clinicopathologic features improves patient stratification for multimodal treatment of gastric cancer
}

\author{
In Cho ${ }^{1,2}$, In Gyu Kwon ${ }^{1,3}$, Ali Guner ${ }^{4}$, Taeil Son ${ }^{5,6}$, Hyoung-Il Kim ${ }^{5,6}$, Dae Ryong \\ Kang $^{7}$, Sung Hoon Noh ${ }^{5,6}$, Joon Seok Lim ${ }^{8}$ and Woo Jin Hyung ${ }^{1,5,6,7,9}$ \\ ${ }^{1}$ Department of Surgery, Graduate School, Yonsei University College of Medicine, Seoul, Republic of Korea \\ ${ }^{2}$ Department of Surgery, Soonchunhyang University College of Medicine, Bucheon, Republic of Korea \\ ${ }^{3}$ Department of Surgery, Keimyung University School of Medicine, Daegu, Republic of Korea \\ ${ }^{4}$ Department of General Surgery, Karadeniz Technical University, Trabzon, Turkey \\ ${ }^{5}$ Department of Surgery, Yonsei University College of Medicine, Seoul, Republic of Korea \\ ${ }^{6}$ Gastric Cancer Center, Yonsei Cancer Center, Yonsei University Health System, Seoul, Republic of Korea \\ 7 Department of Humanities and Social Medicine, Ajou University School of Medicine, Suwon, Republic of Korea \\ ${ }^{8}$ Department of Diagnostic Radiology, Yonsei University College of Medicine, Seoul, Republic of Korea \\ ${ }^{9}$ Robot and MIS Center, Severance Hospital, Yonsei University Health System, Seoul, Republic of Korea \\ Correspondence to: Woo Jin Hyung, email: wjhyung@yuhs.ac \\ Keywords: gastric cancer, preoperative staging, diagnostic accuracy, patient stratification, multimodal treatment \\ Received: December 05, $2016 \quad$ Accepted: May 12, 2017 \\ Published: June 22, 2017
}

Copyright: Cho et al. This is an open-access article distributed under the terms of the Creative Commons Attribution License 3.0 (CC BY 3.0), which permits unrestricted use, distribution, and reproduction in any medium, provided the original author and source are credited.

\section{ABSTRACT}

Preoperative staging of gastric cancer with computed tomography alone exhibits poor diagnostic accuracy, which may lead to improper treatment decisions. We developed novel patient stratification criteria to select appropriate treatments for gastric cancer patients based on preoperative staging and clinicopathologic features. A total of $\mathbf{5 3 5 2}$ consecutive patients who underwent gastrectomy for gastric cancer were evaluated. Preoperative stages were determined according to depth of invasion and nodal involvement on computed tomography. Logistic regression analysis was used to identify clinicopathological factors associated with the likelihood of proper patient stratification. The diagnostic accuracies of computed tomography scans for depth of invasion and nodal involvement were $67.1 \%$ and $74.1 \%$, respectively. Among clinicopathologic factors, differentiated tumor histology, tumors smaller than $\mathbf{5} \mathrm{cm}$, and gross appearance of early gastric cancer on endoscopy were shown to be related to a more advanced stage of disease on preoperative computed tomography imaging than actual pathological stage. Additional consideration of undifferentiated histology, tumors larger than $\mathbf{5} \mathbf{~ c m}$, and grossly advanced gastric cancer on endoscopy increased the probability of selecting appropriate treatment from $75.5 \%$ to $94.4 \%$. The addition of histology, tumor size, and endoscopic findings to preoperative staging improves patient stratification for more appropriate treatment of gastric cancer.

\section{INTRODUCTION}

A global health problem, gastric cancer ranks as the third most common cause of cancer-related deaths worldwide [1]. Despite efforts to improve early detection of gastric cancer by mass screening in Korea and Japan, the proportion of advanced gastric cancers still remains high at $39.2 \%-51.4 \%$ [2-4]. Indeed, the incidence of advanced gastric cancer is much higher in Western countries $[2,5]$. Consequently, developing treatment strategies for patients with advanced gastric cancer has emerged as a major concern, and multimodal treatments, such as neoadjuvant chemotherapy and perioperative or preoperative chemoradiotherapy, have been applied for the treatment of advanced gastric cancer. 
Major guidelines for gastric cancer treatment recommend that decisions are to be made based on clinical staging, primarily achieved with the use of computed tomography (CT) [6-8]. With advances in equipment, development of contrast materials, and applications of stomach cancer-specific protocols, the diagnostic accuracy of CT scans for gastric cancer has improved considerably. The overall diagnostic accuracies of CT scans in predicting tumor depth and nodal status range from $71.4 \%$ to $88.9 \%$ and $75.9 \%$ to $78 \%$, respectively [9-12]. Nevertheless, such accuracies are insufficient for proper treatment decisions. Moreover, studies on preoperative, multimodal treatments have included patients with pathologically early stage gastric cancer who do not require any perioperative or preoperative chemotherapy.

Although many studies have evaluated the accuracy of clinical staging using CT scans for gastric cancer, most have analyzed its accuracy in regards to depth of invasion or nodal status separately. Few studies have focused on evaluating diagnostic accuracy in relation to overall clinical staging. Furthermore, no study has attempted to identify risk factors for inappropriate treatment decisions caused by inaccurate clinical staging, and no study has evaluated clinicopathologic features that may help improve patient stratification for proper decision making.

In the present study, we hypothesized that additional consideration of clinicopathologic features in clinical staging would facilitate proper patient stratification for gastric cancer treatment. Accordingly, we compared preoperative clinical stages to their actual pathologic stages to investigate the diagnostic accuracy of clinical staging with $\mathrm{CT}$ alone in gastric cancer and then evaluated which clinicopathologic features improved the diagnostic accuracy thereof. Additionally, we attempted to develop novel patient stratification criteria to select appropriate treatment modalities for gastric cancer patients based on preoperative staging and clinicopathologic factors found to be associated with improved diagnostic accuracy.

\section{RESULTS}

\section{Patient characteristics}

Patient demographics and clinicopathological characteristics are summarized in Table 1. There were 3491 men and 1861 women in the current study. The mean age was $57.9 \pm 11.9$ years. The mean tumor size was $3.6 \pm 2.7 \mathrm{~cm}$. The tumors were located mainly in the lower third (58.8\%) and lesser curvature (43.5\%) of the stomach. Histologically, undifferentiated tumors were predominant $(56.6 \%)$, compared to differentiated ones (43.4\%). In preoperative clinical staging based on analysis of CT images, the majority of patients were classified as clinical T1 stage (52.3\%) and clinical N- stage (69.4\%). On pathological staging, T1 (56.3\%) and N-(64.5\%) tumors were most common.

\section{Accuracy of preoperative staging}

A comparison of $\mathrm{T}$ classification between the preoperative CT scans and final pathologic results is shown in Table 2. The accuracy of $\mathrm{T}$ classification was

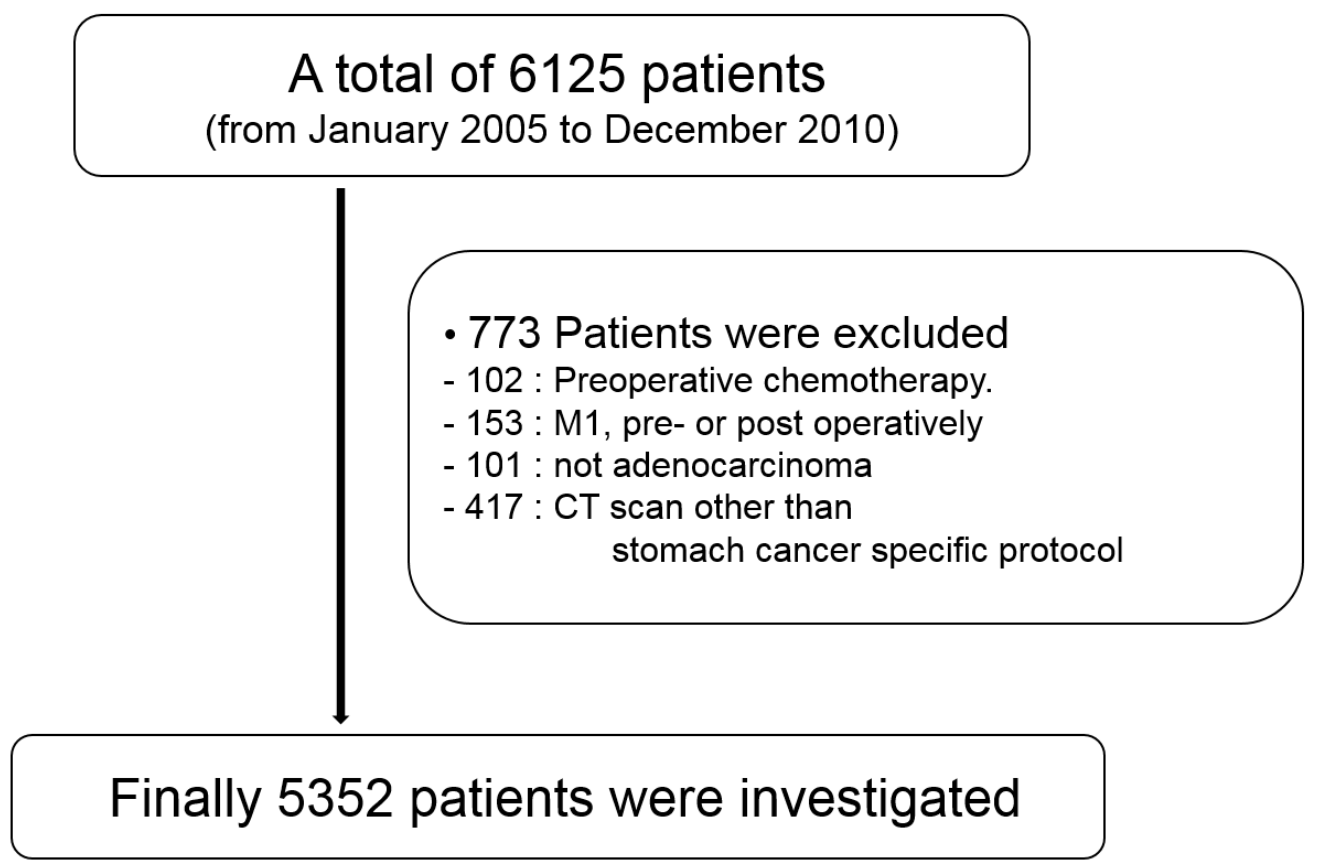

Figure 1: Flow diagram of study patients. 
Table 1: Demographics and clinicopathological features.

\begin{tabular}{|c|c|c|}
\hline Variable & $N=5352$ & $\%$ \\
\hline Age (years) & $57.9 \pm 11.9$ & \\
\hline \multicolumn{3}{|l|}{ Gender } \\
\hline Male & 3491 & 65.2 \\
\hline Female & 1861 & 34.8 \\
\hline Body mass index (kg/m2) & $23.3 \pm 2.9$ & \\
\hline Tumor size (cm) & $3.64 \pm 2.65$ & \\
\hline \multicolumn{3}{|l|}{ Gross finding } \\
\hline EGC & 3046 & 56.9 \\
\hline $\mathrm{AGC}$ & 2306 & 43.1 \\
\hline \multicolumn{3}{|l|}{ Ulceration } \\
\hline Absent & 1910 & 35.7 \\
\hline Present & 3442 & 64.3 \\
\hline \multicolumn{3}{|l|}{ Histology } \\
\hline Differentiated & 2324 & 43.4 \\
\hline Undifferentiated & 3028 & 56.6 \\
\hline \multicolumn{3}{|l|}{ Location, circular } \\
\hline Lesser curvature & 2329 & 43.5 \\
\hline Greater curvature & 735 & 13.7 \\
\hline Anterior wall & 1061 & 19.8 \\
\hline Posterior wall & 1178 & 22.0 \\
\hline Encircling & 49 & 0.9 \\
\hline \multicolumn{3}{|l|}{ Location, tubular } \\
\hline Upper & 682 & 12.7 \\
\hline Middle & 1514 & 28.3 \\
\hline Lower & 3147 & 58.8 \\
\hline Entire & 9 & 0.2 \\
\hline
\end{tabular}

EGC, early gastric cancer; AGC, advanced gastric cancer

Table 2: Correlation of clinical and pathologic stage for depth of invasion and nodal status.

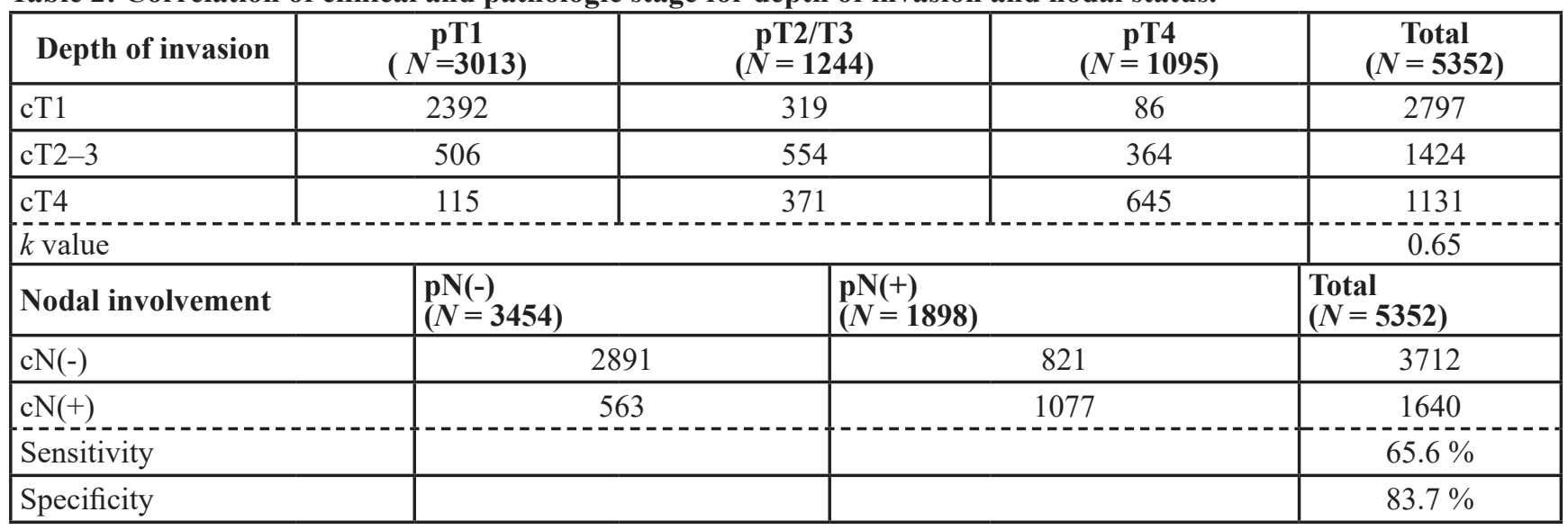

pT, pathological T staging; cT, clinical T staging; pN, pathological N staging; cN, clinical N staging; pT/N, overall pathological staging; cT/N, overall clinical staging. 
Table 3: Correlation of overall clinical and pathologic stage.

\begin{tabular}{|c|c|c|c|c|c|c|c|}
\hline Stage grouping & $\begin{array}{l}\text { pT1/N(-) } \\
(N=2700)\end{array}$ & $\begin{array}{l}\text { pT1/N(+) } \\
(N=313)\end{array}$ & $\begin{array}{l}\text { pT2-3/N(-) } \\
(N=565)\end{array}$ & $\begin{array}{l}\text { pT2-3/N(+) } \\
(N=679)\end{array}$ & $\begin{array}{l}\text { pT4/N(-) } \\
(N=189)\end{array}$ & $\begin{array}{l}\text { pT4/N(+) } \\
(N=906)\end{array}$ & $\begin{array}{l}\text { Total } \\
(N=5352)\end{array}$ \\
\hline cT1/N(-) & 2017 & 166 & 168 & 86 & 26 & 38 & 2501 \\
\hline cT1/N(+) & 175 & 34 & 31 & 34 & 5 & 17 & 296 \\
\hline cT2-3/N(-) & 338 & 54 & 192 & 148 & 46 & 131 & 909 \\
\hline $\mathrm{cT} 2-3 / \mathrm{N}(+)$ & 78 & 36 & 67 & 147 & 36 & 151 & 515 \\
\hline cT4/N(-) & 36 & 6 & 39 & 56 & 29 & 136 & 302 \\
\hline cT4/N(+) & 56 & 17 & 68 & 208 & 47 & 433 & 829 \\
\hline
\end{tabular}

pT, pathological T staging; cT, clinical T staging; pN, pathological N staging; cN, clinical N staging; pT/N, overall pathological staging; cT/N, overall clinical staging.

$67.1 \%$, with overestimation occurring in $18.5 \%$ of patients and underestimation occurring in $14.4 \%$ of patients. The reliability for tumor depth was $k=0.65$. The cT2-3 classification subgroup showed the highest percentage of overestimation $(35.5 \%, 506 / 1424)$. The accuracy of preoperative $\mathrm{N}$ classification was $74.1 \%$ (3968/5352). Overestimation and underestimation of $\mathrm{N}$ classification reached $10.5 \%(563 / 5352)$ and $15.4 \%$ (821/5352), respectively. The sensitivity and specificity for clinical node involvement $(\mathrm{cN}+)$ were $65.6 \%$ and $83.7 \%$, respectively. A comparison between preoperative and postoperative staging is shown in Table 3. The accuracy of overall staging was $53.3 \%$; overestimation and underestimation reached $23.9 \%$ and $22.8 \%$, respectively. The reliability for overall staging was $k=0.69$.

In multivariate analysis of the $\mathrm{cApE}$ and $\mathrm{cApA}$ groups, undifferentiated histology $(p<.001)$, tumors larger than $5 \mathrm{~cm}(p<.001)$, and grossly advanced gastric cancer (AGC) appearance on endoscopy $(p<.001)$ were independently associated with a higher probability of proper patient stratification for treatment (cApA group) (Table 4).

\section{Probability for proper patient stratification}

The probability of proper patient stratification was $75.5 \%$, calculated as the number of patients in the cApA group $(n=1243)$ divided by the number in both the cApE and cApA groups $(n=1646)$ (Table 5). When we additionally considered the clinicopathological features found to be related to diagnostic accuracy in our study,

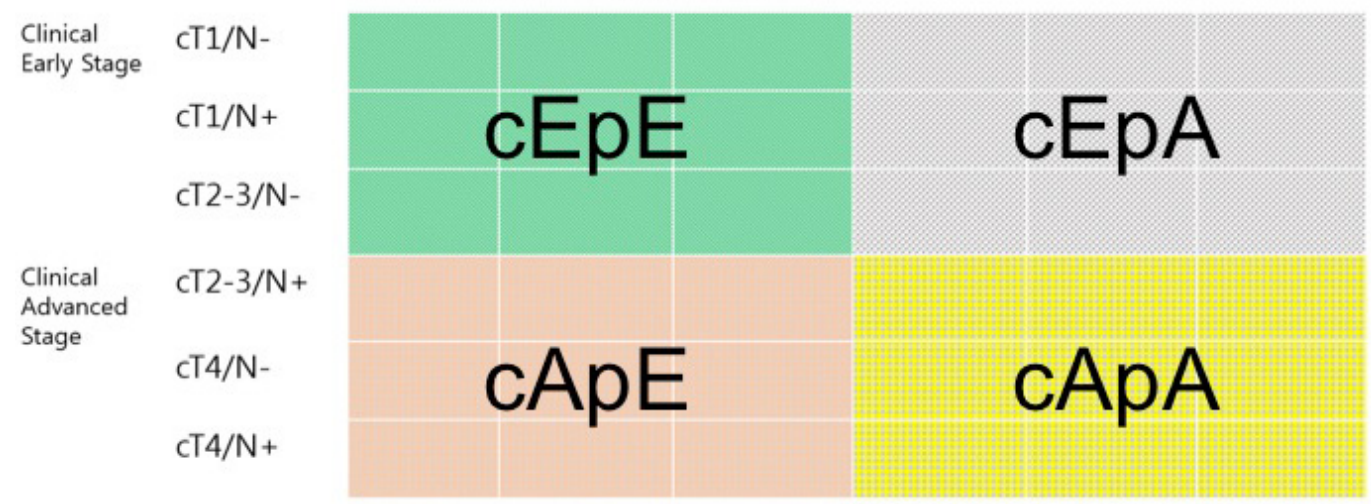

Figure 2: Classification of patients to establish proper selection criteria for treatment modalities. The clinical early and pathological early stage (cEpE) group (green color), the clinical early and pathological advanced stage (cEpA) group (gray color), the clinical advanced and pathological early stage (cApE) group (brown color), and the clinical advanced and pathological advanced stage (cApA) group (yellow color). 
Table 4: Clinicopathological features related to probability of proper patient stratification.

\begin{tabular}{|c|c|c|c|c|c|c|}
\hline & & & Univariate ana & & Multivariate ana & \\
\hline & $(N=403)$ & $(N=1243)$ & OR $(95 \% \mathrm{CI})$ & $P$ & OR $(95 \% \mathrm{CI})$ & $P$ \\
\hline \multicolumn{7}{|l|}{ Gender } \\
\hline Male & 285 & 854 & Ref. & & Ref. & \\
\hline Female & 118 & 389 & $0.91(0.71-1.16)$ & 0.447 & $0.92(0.67-1.27)$ & 0.619 \\
\hline Age (years) & 403 & 1243 & $1.00(0.99-1.01)$ & 0.462 & $1.00(0.99-1.01)$ & 0.929 \\
\hline $\mathrm{BMI}\left(\mathrm{kg} / \mathrm{m}^{2}\right)$ & 403 & 1243 & $1.07(1.03-1.11)$ & $<0.001$ & $1.05(1.01-1.10)$ & 0.029 \\
\hline \multicolumn{7}{|l|}{ Histology } \\
\hline Differentiated & 207 & 396 & Ref. & & Ref. & \\
\hline Undifferentiated & 196 & 847 & $0.44(0.35-0.56)$ & $<0.001$ & $0.54(0.40-0.73)$ & $<0.001$ \\
\hline \multicolumn{7}{|l|}{ Tumor size (cm) } \\
\hline$\leq 5$ & 315 & 505 & Ref. & & Ref. & \\
\hline$>5$ & 88 & 738 & $0.19(0.15-0.25)$ & $<0.001$ & $0.31(0.23-0.42)$ & $<0.001$ \\
\hline \multicolumn{7}{|l|}{ Gross findings } \\
\hline EGC & 192 & 39 & Ref. & & Ref. & \\
\hline AGC & 211 & 1204 & $0.03(0.02-0.05)$ & $<0.001$ & $0.04(0.03-0.07)$ & $<0.001$ \\
\hline \multicolumn{7}{|l|}{ Ulcerative } \\
\hline No & 113 & 242 & Ref. & & Ref. & \\
\hline Yes & 290 & 1001 & $0.62(0.48-0.80)$ & $<0.001$ & $1.124(0.76-1.67)$ & 0.559 \\
\hline \multicolumn{7}{|l|}{ Location, circular } \\
\hline Lesser curvature & 203 & 672 & Ref. & & Ref. & \\
\hline Greater curvature & 49 & 176 & $0.92(0.65-1.31)$ & 0.651 & $0.74(0.46-1.17)$ & 0.198 \\
\hline Anterior wall & 68 & 168 & $1.34(0.97-1.85)$ & 0.075 & $1.23(0.82-1.83)$ & 0.315 \\
\hline Posterior wall & 81 & 192 & $1.40(1.03-1.89)$ & 0.031 & $1.14(0.77-1.68)$ & 0.525 \\
\hline Encircling & 2 & 35 & $0.19(0.05-0.79)$ & 0.023 & $0.36(0.07-1.73)$ & 0.202 \\
\hline \multicolumn{7}{|l|}{ Location, tubular } \\
\hline Upper & 66 & 197 & Ref. & & Ref. & \\
\hline Middle & 102 & 332 & $0.92(0.64-1.31)$ & 0.634 & $0.78(0.50-1.22)$ & 0.280 \\
\hline Lower & 235 & 707 & $0.99(0.72-1.36)$ & 0.961 & $0.75(0.51-1.12)$ & 0.156 \\
\hline Entire & 0 & 7 & NA & NA & NA & NA \\
\hline
\end{tabular}

OR, odds ratio; EGC, early gastric cancer; AGC, advanced gastric cancer.

A*: Clinical early advancedwith pathological advanced early group.

$\mathrm{B}^{\dagger}$ : Clinical advanced with pathological advanced group.

the probability of proper patient stratification greatly improved. By considering only one additional feature (histology, size, or gross appearance), the probability of proper patient stratification improved from $75.5 \%$ to $81.2 \%-89.3 \%$. Moreover, if two variables were considered, the probability increased to $88.0 \%-92.5 \%$. Among patients with undifferentiated histology, tumors larger than $5 \mathrm{~cm}$, and gross AGC appearance on endoscopy, the probability of proper patient stratification improved to $94.4 \%$ : in other words, candidates receiving potentially unnecessary neoadjuvant chemotherapy could be decreased from 403 patients (403/5352) to 30 patients (30/5352). Meanwhile, the probability of proper patient stratification was only $10.5 \%$ in patients who had differentiated histology, tumors smaller than $5 \mathrm{~cm}$, and early gastric cancer (EGC) appearance on endoscopy.

\section{DISCUSSION}

In the present study, the accuracies of estimating depth of invasion and nodal involvement on CT scans alone were $67.1 \%$ and $74.1 \%$, respectively. The accuracy of overall staging was only $53.3 \%$. More specifically, $35.5 \%$ and $34.3 \%$ of patients were overestimated for clinical T2-3 classification and node positivity, respectively. When we additionally considered the clinicopathologic features identified in our study to be associated with proper patient stratification, the probability thereof improved from $75.5 \%$ to $94.4 \%$.

When deciding on a treatment modality before 
Table 5: Probability of proper patient stratification when considering clinicopathological features.

\begin{tabular}{|c|c|c|c|}
\hline (N) & \begin{tabular}{|l|}
$\begin{array}{l}\text { Clinical advanced and } \\
\text { pathological early group }\end{array}$ \\
\end{tabular} & \begin{tabular}{|l|}
$\begin{array}{l}\text { Clinical advanced and pathological } \\
\text { advanced group }\end{array}$ \\
\end{tabular} & Probability $(\%)$ \\
\hline Variable not considered & \begin{tabular}{|l|}
403 \\
\end{tabular} & 1243 & 75.5 \\
\hline \multicolumn{4}{|l|}{ One variable considered } \\
\hline $\mathrm{AGC}^{\S}$ & 211 & 1204 & 85.1 \\
\hline$>5 \mathrm{~cm}^{*}$ & 88 & 738 & 89.3 \\
\hline Undiff $^{\dagger}$ & 196 & 847 & 81.2 \\
\hline \multicolumn{4}{|l|}{ Two variables considered } \\
\hline $\mathrm{AGC}^{\S}+>5 \mathrm{~cm}^{*}$ & 59 & 724 & 92.5 \\
\hline$>5 \mathrm{~cm}^{*}+$ Undiff $^{+}$ & 46 & 520 & 91.9 \\
\hline Undiff $^{\dagger}+$ AGC $^{\S}$ & 111 & 820 & 88.0 \\
\hline \multicolumn{4}{|l|}{ Three variables considered } \\
\hline $\mathrm{AGC}^{\S}+>5 \mathrm{~cm}^{*}+$ Undiff $^{\dagger}$ & 30 & 507 & 94.4 \\
\hline
\end{tabular}

AGC§: grossly advanced gastric cancer appearance in endoscopy; $>5 \mathrm{~cm} *$ : tumor size larger than $5 \mathrm{~cm}$; Undiff : undifferentiated histology.

surgery, physicians must take into account the integrated clinical stage of each patient; however, preoperative staging based on $\mathrm{CT}$ scans alone can be inaccurate. Nevertheless, few studies have attempted to evaluate the accuracy of overall clinical staging using CT scans alone. In the current study, we found that the accuracy thereof in gastric cancer patients was only $53.3 \%$.

Treatment modalities are commonly selected according to clinical stage. Japanese gastric cancer treatment guidelines state that there is no established benefit of neoadjuvant chemotherapy for gastric cancer patients. Meanwhile, Western guidelines recommend neoadjuvant chemotherapy or surgery for patients with stage II disease or higher (cT2-3N+ or cT4N-/+) [13]. The present study, however, found that the probability of delivering inappropriate treatment was $24.5 \%$ when relying on CT scans alone to conduct preoperative clinical staging. When we considered clinicopathologic features of histology, tumor size, and endoscopic findings, the probability of delivering an inappropriate treatment modality decreased to $5.6 \%$.

Our results indicate that undifferentiated histology, tumors larger than $5 \mathrm{~cm}$, and grossly AGC appearance on endoscopy are independently associated with a higher probability of reaching a proper treatment decision. To the best of our knowledge, this study is the first to evaluate clinicopathologic features that can improve stratification of patients with gastric cancer. When we applied the identified clinicopathologic features, the likelihood of proper patient stratification increased from $75.5 \%$ to $94.4 \%$. Therefore, by considering these clinicopathologic features, physicians can spare patients with early staged tumors, who can be cured by surgery alone, from unnecessary pretreatment.

Neoadjuvant chemotherapy has been commonly used to treat resectable gastric cancer in Western countries. Its application is based on two large phase III clinical trials $[13,14]$, which demonstrated that perioperative chemotherapy facilitated significant improvements in resectability, disease-free survival, and overall survival, compared to surgery alone. However, despite these favorable results, the studies were critically limited in that no standard clinical staging method was applied at the time of randomization. As a result, patients in the surgeryonly group with $\mathrm{pT} 1$ and $\mathrm{pN}$ - classification comprised $8.3 \%$ and $26.9 \%$, respectively, of patients in the MAGIC trial [13]. Also, in the FNCLCC/FFCD trial [14], patients with a pN- classification accounted for $20 \%$ of the surgeryonly group $[13,14]$. This means that similar proportions of patients that did not need chemotherapy were included in the perioperative chemotherapy groups in those studies. Accordingly, physicians should caution against applying unnecessary neoadjuvant chemotherapy to early stage patients, as doing so introduces greater medical expense, causes suffering due to serious side effects, and postpones the timing of surgery. By applying the clinicopathologic risk factors shown to be significant for proper patient satisfaction in the current study, which are readily collected during routine patient evaluations, physicians can protect their patients from unnecessary neoadjuvant chemotherapy. In our study, the number of candidates receiving unnecessary neoadjuvant chemotherapy could have been decreased from 403 to 30 patients among 5352 patients.

This study is superior to previous studies for the following reasons: First, we utilized data from a largescale database collected over a relatively short period. Additionally, CT scans and resected specimens were evaluated, respectively, by radiologists and pathologists specializing in upper gastroenterology at a single, high-volume center. As well, preoperative staging was evaluated using CT scans acquired following a stomach cancer-specific protocol. Therefore, the data were of similar quality and highly reliable. 
Notwithstanding, there are several limitations to the current study that warrant consideration. First, there may be bias in our study due to the retrospective nature of the analyses, although we used a large-scale, homogenous database. First, there may be bias in our study due to the retrospective nature of the analyses, although we used a large-scale, homogenous database. Additionally, our use of two different gastric distension methods could have affected the evaluation of clinical T classification, and the possibility of inter-radiologist differences in evaluation of CT images could not be excluded, although we used the same criteria for clinical $\mathrm{T}$ and $\mathrm{N}$ classification. Therefore, bias in deciding clinical $\mathrm{T}$ and $\mathrm{N}$ factors could not be completely avoided. Moreover, since we excluded patients who received neoadjuvant chemotherapy, bias related to this exclusion might be present in the analysis cApE and cApA patients. Second, we did not consider other diagnostic modalities, such as diagnostic laparoscopy and endoscopic ultrasound. If additional diagnostic tools were included for preoperative staging, diagnostic accuracy may have been increased. Nevertheless, CT scans are non-invasive and are currently regarded as a standard staging method. It is, therefore, worth noting that a simple combination of CT scans and clinicopathologic features improves patient stratification. Third, the preoperative nodal staging system utilized in this study differed from the AJCC pathologic staging system. Although we recognized differences between the two systems, we were unable to take them into account due to the retrospective design of our study. Further prospective studies with various diagnostic modalities are necessary to validate our findings. Fourth, using the three factors we have identified, underdiagnosis would likely be increased. However, surgery with adjuvant chemotherapy would not be considered undertreatment, rather it is a standard treatment option for stage II or III gastric cancer patients. Since the purpose of this study was to develop novel stratification criteria with which to select proper gastric cancer patients for neoadjuvant chemotherapy, we have focused on clinically advanced cancers. Lastly, due to the retrospective nature of the study, we discerned histology from pathologic evaluation of resected specimens. Thus, the possibility of changes in histologic type after resection must be considered, although discrepancies in histologic diagnosis between biopsy and pathologies of resected specimens reportedly range only from $2.4 \%$ to $4.4 \%$ [1517].

Current practices for treating gastric cancer based on preoperative staging with $\mathrm{CT}$ scans alone are associated with a high probability of improper treatment decisions due to low diagnostic accuracy. By additionally considering clinicopathologic features of histology, tumor size, and endoscopic findings, more appropriate treatment strategies can be established.

\section{MATERIALS AND METHODS}

\section{Patients and clinicopathological features}

A retrospective review of a prospectively maintained database for gastric cancer revealed a total of 6125 consecutive patients who underwent gastrectomy for primary gastric cancer from January 2005 to December 2010. We excluded patients from the analysis who fulfilled the following criteria: (1) received neoadjuvant therapy $(n=102)$; (2) received a preoperative or postoperative diagnosis of distant metastasis $(n=153)$; (3) did nothave adenocarcinoma ( $n=101)$; and (4) were not examined with CT following a stomach cancer-specific protocol ( $n$ =417). Finally, 5352 patients were included for analysis. (Figure 1) This study was reviewed and approved by the Institutional Review Board of Severance Hospital, Yonsei University Health System (4-2013-0510).

The demographics of the patients, including age, sex, and pathological features of histology, size, location, presence of ulceration, and gross type on endoscopy, were evaluated. Histologic types were classified into two groups: differentiated, which included papillary, well-differentiated, or moderately-differentiated adenocarcinoma, and undifferentiated, which included poorly-differentiated or undifferentiated adenocarcinoma, signet ring cell carcinoma, and mucinous carcinoma. Gross types were classified into two groups based on endoscopic finings as EGC or AGC [18].

\section{Stomach cancer-specific CT protocol}

Preoperative staging was conducted based on CT scans taken in accordance with a stomach cancer-specific protocol. The equipment was a 16- or 64-detector row CT scan (Sensation 16 or 64; Siemens Medical Solutions, Germany). Each patient fasted for 4 hours before the CT examination. Before the CT scan, patients were injected with 10mg of butylscopolamine bromide (Buscopan; BoehringerIngelheim, Germany) to minimize bowel peristalsis and to induce hypotonia. Images were acquired from the level of the diaphragm to the symphysis pubis with detector collimations of $16 \times 0.75 \mathrm{~mm}$ or $64 \times 0.6 \mathrm{~mm}$. Other scanning parameters were as follows: $160 \mathrm{mAs}$; $120 \mathrm{kVp}$; table speed of $24 \mathrm{~mm}$ per rotation; and gantry rotation time of 0.5 seconds. For gastric distention, we initially used water as an oral contrast agent (800$1000 \mathrm{ml}$ ). Beginning in January 2007, we used two packs of effervescent granules for gastric distention. CT scans were obtained with the patient in the supine position irrelevant to the ingested oral contrast. All patients received $120-150 \mathrm{ml}$ of contrast medium intravenously using an automatic injector at a rate of 3-4ml/s. Images were acquired in the arterial and portal phases. Axial and 
coronal images were reconstructed with a 3-mm thickness section and a 3-mm interval.

\section{Preoperative CT evaluation}

The CT scans were evaluated by radiologists specialized in upper gastrointestinal imaging. Mural invasion of gastric cancer into the gastric wall was classified according to previously published criteria [1922]. A cT1 lesion was defined as non-visualization of an abnormal mural lesion or mucosal thickening with enhancement (cT1a) or visualization of a low attenuation stripe at the base of the lesion, corresponding to the submucosal layer (cT1b). A cT2-3lesion was defined as a thickening of the gastric wall with a loss or disruption of a low attenuating stripe and a clear and smooth outer gastric surface around the lesion. Since CT criteria for cT3 (subserosal layer) lesions have not been established and the serosal layer of the gastric wall is not visible on CT images, differentiation of cT2 (proper muscle layer) from cT3 lesions on CT images is nearly impossible. Thus, we defined cT2 and cT3 lesions as cT2-3. All cT4a lesions demonstrated nodular or irregular outer borders of a thickened gastric wall or perigastric fat infiltration, and all cT4b lesions showed the changes described for cT4a lesions in addition to extension into adjacent organs. Positive lymph nodes were identified on the basis of a size larger than $8 \mathrm{~mm}$ along the short axis [23]. According to this criterion, nodal status on CT scans was classified as follows: $\mathrm{cN}$-, no significant lymph node enlargement, or $\mathrm{cN}+$, lymph node enlargement.

\section{Pathologic staging}

Pathologic evaluation of depth of invasion was performed with resected surgical specimens based on the 7th edition of the International Union Against Cancer Classification (AJCC) as follows: pT1, tumor invasion of the mucosa and/or submucosa; $\mathrm{pT} 2$, tumor invasion of the muscularis propria; $\mathrm{pT} 3$, tumor invasion of the subserosa; pT4a, tumor penetration of the serosa; and pT4b, tumor invasion of adjacent structures [22, 24]. Lymph nodes were categorized as $\mathrm{pN}$-, no lymph node metastasis, or $\mathrm{pN}+$, more than one lymph node metastasis.

\section{Grouping of patients}

Although treatment modalities were decided by clinical staging, the appropriateness of the treatment decisions was assessed according to pathologic stage. To establish proper selection criteria for treatment modalities, we grouped patients into one of four groups (Figure 2): (1) the clinical early and pathological early stage (cEpE) group, including clinical stages cT1/N-, cT1/N+, or cT2-
$3 / \mathrm{N}$ - and pathological stages $\mathrm{pT} 1 / \mathrm{N}-, \mathrm{pT} 1 / \mathrm{N}+$, or $\mathrm{pT} 2-$ $3 / \mathrm{N}-$; (2) the clinical early and pathological advanced stage (cEpA) group, including clinical stages cT1/N-, $\mathrm{cT} 1 / \mathrm{N}+$, or cT2-3/N- and pathological stages pT2-3/ $\mathrm{N}+$, pT4/N-, or pT4/N+; (3) the clinical advanced and pathological early stage (cApE) group, including clinical stages cT2-3/N+, cT4/N-, or cT4/N+ and pathological stages pT1/N-, pT1/N+, or pT2-3/N-; and (4) the clinical advanced and pathological advanced stage (cApA) group, including clinical stages cT2-3/N+, cT4/N-, or cT4/N+ and pathological stages pT2-3/N+, pT4/N-, or pT4/N+. In general, patients in the cEpE and cEpA groups underwent surgery first. Meanwhile, patients in the cApE and cApA groups underwent either surgery or neoadjuvant/ perioperative treatment. Between the $\mathrm{cApE}$ and $\mathrm{cApA}$ groups, patients in the cApA group were more likely to receive more appropriate treatment with accurate clinical staging, which we considered to reflect proper patient stratification. However, cApE patients may have possibly received inappropriate treatment, since the treatment modality was selected according to inaccurate preoperative clinical staging (i.e., improper patient stratification).

\section{Definition of overestimation and underestimation}

Overestimation of the depth of invasion (T) was defined as a case in which the tumor depth on preoperative evaluation by CT scan was deeper than that on final pathologic evaluation. If the tumor depth on preoperative evaluation by CT scan was shallower than that on final pathologic evaluation, $\mathrm{T}$ was regarded as being underestimated. Similarly, overestimation of nodal involvement $(\mathrm{N})$ was defined as preoperative CT scans showing significant lymph nodes, despite the absence of an involved lymph node on final pathologic evaluation. Underestimation of $\mathrm{N}$ was defined when node-negative gastric cancer on $\mathrm{CT}$ scans exhibited the presence of metastatic lymph nodes in the resected specimen. For overall staging, overestimation and underestimation were defined as preoperative clinical stage that was higher or lower than the pathological stage, respectively.

\section{Statistical analysis}

For statistical analysis, SPSS software (version 18.0; SPSS Inc., Chicago, IL, USA) was used. Categorical variables were analyzed using the chi-square test, and continuous data were analyzed using the Mann-Whitney test. For multivariate analysis, logistic regression analysis was used. All values of $p$ less than 0.05 wereconsidered to demonstrate statistical significance.

We assessed reliability by weighted $k$ [25]. Weighted $k$ was computed to quantify agreement between the CT findings and final pathology. For weighted $k$, full weight was given to perfect agreement; half weight was given 
to disagreement by one grade; and zero weight was given to other disagreements. The degrees of agreement were categorized as follows: values of $0.00-0.20$, poor agreement; values of 0.21-0.40, fair agreement; values of 0.41-0.60, moderate agreement; values of 0.61-0.80, good agreement; and values of 0.81-1.00, excellent agreement [26].

\section{Abbreviations}

CT, computed tomography; pT, pathological T staging; cT, clinical T staging; $\mathrm{pN}$, pathological $\mathrm{N}$ staging; $\mathrm{cN}$, clinical $\mathrm{N}$ staging; $\mathrm{pT} / \mathrm{N}$, overall pathological staging; cT/N, overall clinical staging; EGC, early gastric cancer; $\mathrm{AGC}$, advanced gastric cancer; $\mathrm{cEpE}$, clinical early and pathological early stage; cEpA, the clinical early and pathological advanced stage; cApE, the clinical advanced and pathological early stage; cApA, the clinical advanced and pathological advanced stage

\section{Author contributions}

In Cho, In Gyu Kwon, Hyoung-il Kim, Sung Hoon Noh, and Woo Jin Hyung designed the study. In Cho, In Gyu Kwon, Ali Guner, Taeil Son, Hyoung-il Kim, and Sung Hoon Noh collected the data. DaeRyong Kang, JoonSeok Lim, and Woo Jin Hyung did the statistical analyses. In Cho, In Gyu Kwon, JoonSeok Lim, and Woo Jin Hyung wrote the paper. Ali Guner, TaeilSon, Hyoungil Kim, DaeRyong Kang, Sung Hoon Noh, and JoonSeok Lim critically revised subsequent drafts. All authors read and approved the submitted version.

\section{ACKNOWLEDGMENTS}

We would like to thank Anthony Milliken for his help with editing the manuscript. We acknowledge the assistance of BioScience Writers, LLC (Houston, TX, USA) in copyediting of the manuscript and corrections of English language usage.

\section{CONFLICTS OF INTEREST}

The authors have declared no conflicts of interest.

\section{FUNDING}

This work was supported by a grant from the National R \& D Program for Cancer Control, Ministry of Health \& Welfare, Republic of Korea [grant number 1320270].

\section{REFERENCES}

1. Ferlay J, Soerjomataram I, Dikshit R, Eser S, Mathers C, Rebelo M, Parkin DM, Forman D, Bray F. Cancer incidence and mortality worldwide: sources, methods and major patterns in GLOBOCAN 2012. Int J Cancer. 2015; 136: E359-86. doi: 10.1002/ijc.29210.

2. Strong VE, Song KY, Park CH, Jacks LM, Gonen M, Shah M, Coit DG, Brennan MF. Comparison of gastric cancer survival following R0 resection in the United States and Korea using an internationally validated nomogram. Ann Surg. 2010; 251: 640-6. doi: 10.1097/ SLA.0b013e3181d3d29b.

3. Ahn HS, Lee HJ, Hahn S, Kim WH, Lee KU, Sano T, Edge SB, Yang HK. Evaluation of the seventh American Joint Committee on Cancer/International Union Against Cancer Classification of gastric adenocarcinoma in comparison with the sixth classification. Cancer. 2010; 116: 5592-8. doi: $10.1002 /$ cncr.25550.

4. Jeong O, Park YK. Clinicopathological features and surgical treatment of gastric cancer in South Korea: the results of 2009 nationwide survey on surgically treated gastric cancer patients. J Gastric Cancer. 2011; 11: 69-77. doi: 10.5230/ jgc.2011.11.2.69.

5. Dassen AE, Dikken JL, Bosscha K, Wouters MW, Cats A, van de Velde CJ, Coebergh JW, Lemmens VE. Gastric cancer: decreasing incidence but stable survival in the Netherlands. Acta Oncol. 2014; 53: 138-42. doi: 10.3109/0284186X.2013.789139.

6. Ajani JA, Barthel JS, Bekaii-Saab T, Bentrem DJ, D'Amico TA, Das P, Denlinger C, Fuchs CS, Gerdes H, Hayman JA, Hazard L, Hofstetter WL, Ilson DH, et al. Gastric cancer. J Natl Compr Canc Netw. 2010; 8: 378-409.

7. Okines A, Verheij M, Allum W, Cunningham D, Cervantes A. Gastric cancer: ESMO Clinical Practice Guidelines for diagnosis, treatment and follow-up. Ann Oncol. 2010; 21: v50-4. doi: 10.1093/annonc/mdq164.

8. Japanese Gastric Cancer Association. Japanese gastric cancer treatment guidelines 2010 (ver. 3). Gastric Cancer. 2011; 14: 113-23. doi: 10.1007/s10120-011-0042-4.

9. Chen CY, Hsu JS, Wu DC, Kang WY, Hsieh JS, Jaw TS, Wu MT, Liu GC. Gastric cancer: preoperative local staging with 3D multi-detector row CT--correlation with surgical and histopathologic results. Radiology. 2007; 242: 472-82. doi: 10.1148/radiol.2422051557.

10. Kwee RM, Kwee TC. Imaging in local staging of gastric cancer: a systematic review. J Clin Oncol. 2007; 25: 2107 16. doi: 10.1200/JCO.2006.09.5224.

11. House MG, Brennan MF. Neoadjuvant therapy for gastric cancer. Adv Surg. 2008; 42: 151-68.

12. Hasegawa S, Yoshikawa T, Shirai J, Fujikawa H, Cho H, Doiuchi T, Yoshida T, Sato T, Oshima T, Yukawa N, Rino Y, Masuda M, Tsuburaya A. A prospective validation study to diagnose serosal invasion and nodal metastases of gastric 
cancer by multidetector-row CT. Ann Surg Oncol. 2013; 20: 2016-22. doi: 10.1245/s10434-012-2817-1.

13. Cunningham D, Allum WH, Stenning SP, Thompson JN, Van de Velde CJ, Nicolson M, Scarffe JH, Lofts FJ, Falk SJ, Iveson TJ, Smith DB, Langley RE, Verma M, et al. Perioperative chemotherapy versus surgery alone for resectable gastroesophageal cancer. N Engl J Med. 2006; 355: 11-20. doi: 10.1056/NEJMoa055531.

14. Ychou M, Boige V, Pignon JP, Conroy T, Bouche O, Lebreton G, Ducourtieux M, Bedenne L, Fabre JM, SaintAubert B, Geneve J, Lasser P, Rougier P. Perioperative chemotherapy compared with surgery alone for resectable gastroesophageal adenocarcinoma: an FNCLCC and FFCD multicenter phase III trial. J Clin Oncol. 2011; 29: 1715-21. doi: 10.1200/JCO.2010.33.0597.

15. Kim JM, Sohn JH, Cho MY, Kim WH, Chang HK, Jung ES, Kook MC, Jin SY, Chae YS, Park YS, Kang MS, Kim H, Lee JH, et al. Pre- and post-ESD discrepancies in clinicopathologic criteria in early gastric cancer: the NECAKorea ESD for Early Gastric Cancer Prospective Study (N-Keep). Gastric Cancer. 2016; 19: 1104-13. doi: 10.1007/ s10120-015-0570-4.

16. Takao M, Kakushima N, Takizawa K, Tanaka M, Yamaguchi Y, Matsubayashi H, Kusafuka K, Ono H. Discrepancies in histologic diagnoses of early gastric cancer between biopsy and endoscopic mucosal resection specimens. Gastric Cancer. 2012; 15: 91-6. doi: 10.1007/ s10120-011-0075-8.

17. Shim CN, Kim H, Kim DW, Chung HS, Park JC, Lee H, Shin SK, Lee SK, Lee YC. Clinicopathologic factors and outcomes of histologic discrepancy between differentiated and undifferentiated types after endoscopic resection of early gastric cancer. Surg Endosc. 2014; 28: 2097-105. doi: 10.1007/s00464-014-3441-X.

18. Sano T, Okuyama Y, Kobori O, Shimizu T, Morioka Y. Early gastric cancer. Endoscopic diagnosis of depth of invasion. Dig Dis Sci. 1990; 35: 1340-4.

19. Furukawa K, Miyahara R, Itoh A, Ohmiya N, Hirooka Y, Mori K, Goto H. Diagnosis of the invasion depth of gastric cancer using MDCT with virtual gastroscopy: comparison with staging with endoscopic ultrasound. AJR Am J Roentgenol. 2011; 197: 867-75. doi: 10.2214/AJR.10.5872.

20. Kumano S, Murakami T, Kim T, Hori M, Iannaccone R, Nakata S, Onishi H, Osuga K, Tomoda K, Catalano C, Nakamura H. T staging of gastric cancer: role of multidetector row CT. Radiology. 2005; 237: 961-6. doi: 10.1148/radiol.2373041380.
21. Kim AY, Kim HJ, Ha HK. Gastric cancer by multidetector row CT: preoperative staging. Abdom Imaging. 2005; 30 : 465-72. doi: 10.1007/s00261-004-0273-5.

22. Lee MH, Choi D, Park MJ, Lee MW. Gastric cancer: imaging and staging with MDCT based on the 7th AJCC guidelines. Abdom Imaging. 2012; 37: 531-40. doi: 10.1007/s00261-011-9780-3.

23. Lim JS, Yun MJ, Kim MJ, Hyung WJ, Park MS, Choi JY, Kim TS, Lee JD, Noh SH, Kim KW. CT and PET in stomach cancer: preoperative staging and monitoring of response to therapy. Radiographics. 2006; 26: 143-56. doi: 10.1148/rg.261055078.

24. Washington K. 7th edition of the AJCC cancer staging manual: stomach. Ann Surg Oncol. 2010; 17: 3077-9. doi: 10.1245/s10434-010-1362-z.

25. Cohen J. Weighted kappa: nominal scale agreement with provision for scaled disagreement or partial credit. Psychol Bull. 1968; 70: 213-20.

26. Fleiss J. Statistical Methods for Rates and Proportions. 2nd ed New York, NY: John Wiley \& Sons. 1981: 212-36. 